\title{
NOTAS SOBRE LAS FUENTES GRIEGAS DE LA EDICIÓN ALDINA \\ DE LA BIBLIA (Y DE LA POLÍGLOTA COMPLUTENSE) \\ Y EL MANUSCRITO UCM 22 (= 442 RAHLFS): \\ A PROPÓSITO DE JUECES 5.26-30
}

\author{
Felipe G. Hernández Muñoz \\ Universidad Complutense de Madrid \\ fhmunoz@ucm.es
}

\section{RESUMEN}

Se estudian las fuentes griegas de la edición Aldina y de la Biblia Políglota Complutense y su relación con el manuscrito UCM 22 en varios pasajes de Jueces (5.26-30).

Palabras Clave: Biblia, Jueces, edición, Aldina, Políglota, fuentes, manuscrito UCM 22.

NOTES ON THE GREEK SOURCES OF THE BIBLE ALDINA EDITION

(AND OF THE POLÍGLOTA COMPLUTENSE) AND THE UCM 22 (= 442 RAHLFS) MANUSCRIPT:

JUDGES 5.26-30

\section{ABSTRACT}

The Greek sources of the Aldina edition and of the Biblia Políglota Complutense and their relationship with the UCM 22 manuscript are studied in several passages of Judges.

KeYwords: Bible, Judges, edition, Aldina, Políglota, sources, UCM 22 manuscript.

La cuestión de las fuentes de la edición Aldina de la Biblia griega (Septuaginta) dista todavía de haber sido aclarada definitivamente ${ }^{1}$. A la probable existencia de varios modelos, se añade el hecho de cambios de fuentes según estemos en uno u otro libro bíblico e, incluso, dentro de un mismo libro, además de ciertas intervenciones de los editores en el texto. La reciente digitalización de los fragmentos conservados del manuscrito griego UCM 22 (= 442 Rahlfs, copiado, al parecer, en Venecia, a comienzos del s. XVI) ${ }^{2}$, fuente de la Políglota de Alcalá (edición casi contemporánea de la Aldi$\left.\mathrm{na}^{3}\right)$, que durante mucho tiempo se dio por perdido durante los combates en la guerra civil española en la Ciudad Universitaria ${ }^{4}$, puede proyectar nuevas luces a la cuestión, máxime cuando dicho manuscrito fue anotado por Marcos Musuro, quien también anotó, aunque más escasamente, el también contemporáneo manuscrito Lond. 10968, considerado fuente de la edición Aldina ${ }^{5}$ y con el que el manuscrito Complutense parece tener estrecha relación textual, quizá por compartir un mismo modelo, el Marc. 5.

Para este merecido homenaje a nuestro querido colega Ángel Martínez Fernández presentamos el testimonio de la Aldina, la Políglota, el manuscrito UCM 22 (y otros testimonios relacionados) en tres pasajes del libro de Jueces que pueden ilustrar 
la complejidad de la cuestión. Aunque en los fragmentos hoy conservados del UCM 22 faltan estos pasajes de Jueces, disponemos de la transcripción que en su día P. de Gayangos, bibliotecario de la Complutense, realizó para F. Delitzsch $c a$. 1886, cuando el manuscrito todavía estaba completo, que podemos utilizar instar codicis.

Se trata de tres textos en un pasaje problemático desde el punto de vista textual dentro del llamado "Canto de Débora", 5.26-30, en el que hemos podido colacionar manuscritos que lo transmiten actualmente, entre ellos los Marcianos (2, 3, 4, 5 y 66)

${ }^{1}$ Cf. recientemente Hernández Muñoz (2020: 235, n. 22; 236, n. 23; 245, n. 51; 246, n. 53), quien habla de una cuestión todavía "abierta". Las sospechas parecen centrarse en el Marc. 5 (= 68 Rahlfs), del s. XV, al que se unen otros códices, según los distintos investigadores: los Marc. 2 y Marc. 4 según Delitzsch (1886: 24); los Marc. 1 y Marc. 6 según Revilla (1917: 104); los Marc. 2 y Marc. 3 según Sweete (1902: 174) y Fernández Marcos (2014: 126); el Marc. 6 para Lagarde (1868: 6-7). En nuestro estudio anteriormente citado hemos destacado pasajes en los que Marc. 5 y Aldina se encuentran en grupos textuales distintos. Este trabajo, con conclusiones todavía provisionales, fruto de una investigación in fieri, se ha realizado en el marco del proyecto: «Manuscritos griegos en España y su contexto europeo (III): Estudios históricos, filológicos y crítico-textuales», PID2019-05733GB-I00.

${ }^{2}$ Accesible en: https://ucm.on.worldcat.org/oclc/1025144100.

La digitalización actual, ya casi completa, no habría podido realizarse sin la contribución de otros colegas que nos precedieron con sus trabajos sobre el manuscrito, como Antonio Bravo, Natalio Fernández Marcos, Jesús Ángel, pero, sobre todo, más recientemente, gracias a Carlos de Jesus, con quien pudimos darle el impulso definitivo con el apoyo técnico del personal de la Biblioteca Histórica de la Universidad Complutense (Martins de Jesus, en prensa). A todos ellos rendimos homenaje de sincero agradecimiento, sin olvidarnos de Antonio Revilla, quien fue el último investigador conocido que tuvo la fortuna de manejar el manuscrito completo en 1917. Después de su parcial destrucción durante la guerra civil española (1936-1939), la digitalización que hemos podido preparar constituye, pues, el intento más completo realizado hasta ahora por recuperar el manuscrito en la forma más cercana a la que originalmente tuvo.

${ }^{3} \mathrm{Y}$ con la que parece mantener vínculos textuales, quizá porque Marcos Musuro, anotador del Lond. 10968, uno de los modelos comprobados de la Aldina, pero también del UCM 22, modelo a su vez de la Políglota, pudo ejercer de eslabón común a ambas. De hecho, en un trabajo reciente (Hernández Muñoz, 2020: 231, n.6) hemos sugerido la posibilidad de que ambos manuscritos bíblicos anotados por Musuro, y hasta cierto punto complementarios por su contenido, sirvieran, en principio, para la elaboración de la edición Aldina, en la que Musuro colaboró, y que el UCM 22 fuera luego enviado por el Senado de Venecia al cardenal Cisneros, promotor de la Políglota en Alcalá, como ayuda para los trabajos de esta edición por él promovida.

${ }^{4}$ Cf. De Andrés, 1974: 230-232; Domingo Malvadi, 2014: 278-280; Martínez Manzano Hernández Muñoz (2019: 141-144).

${ }^{5}$ Cf. Cataldi (1998: 451, 458), quien reconoció marcas de impresión en el manuscrito, cf. también Speranzi (2013: 271): ambos manuscritos, Londinense y Complutense, serían los únicos bíblicos anotados por Musuro. Seguramente el Londinense, de copista todavía no identificado con seguridad, pero probablemente realizado también en Venecia a principios del s. XVI, fue "un" modelo de la Aldina, pero no, pace Cataldi, "el" único modelo. Su testimonio no podemos utilizarlo aquí porque también está dañado y actualmente no transmite Jueces. Como ya se ha indicado, no puede descartarse que el UCM 22 también fuera utilizado (o al menos preparado) para la edición Aldina, y luego enviado a Cisneros en Alcalá para la de la Políglota.

${ }^{6}$ El venerable Marc. 1 (= 23 Rahlfs), del s. VIII, no transmite Jueces, por lo que no podemos incluirlo en nuestro estudio, como tampoco el Marc. 16 (=731, probable modelo del Marc. 5), del s. XIII, el Lond. 10968, del XVI in., y el Vatic. 346 (=248), del s. XIII-XIV, que, sin embargo, es fuente 
propuestos hasta ahora como modelos de la Aldina, además de las ediciones principes Aldina y Políglota, así como otros manuscritos considerados modelos de la Políglota, como el Vat. 330, el Paris. 3 y el propio UCM 22 (como queda dicho, en la transcripción de P. Gayangos) .

TEXTO 1 (Jueces, 5.26) ${ }^{7}$

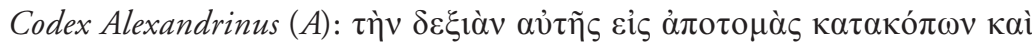

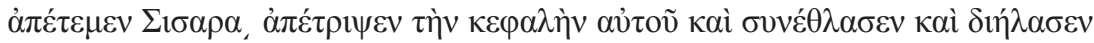

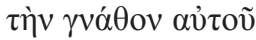

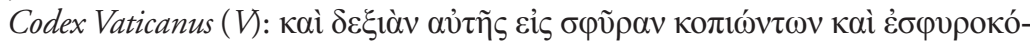

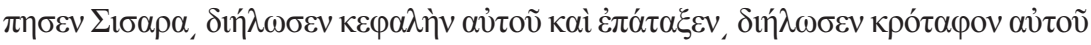

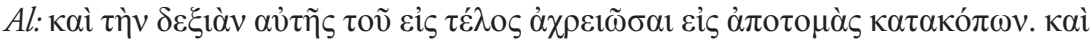

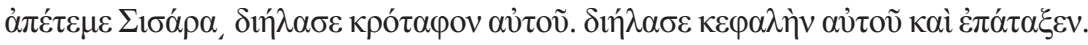

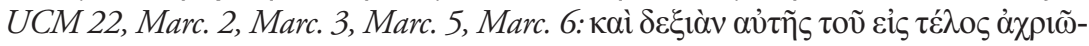

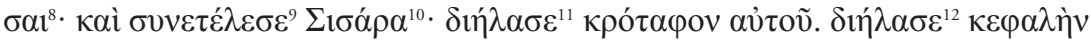

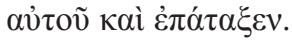

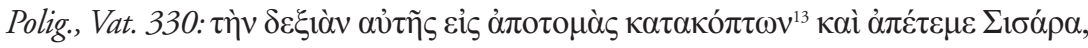

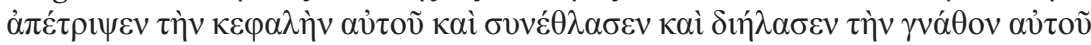

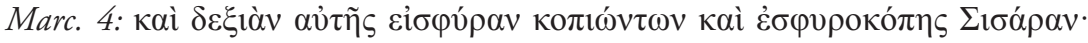

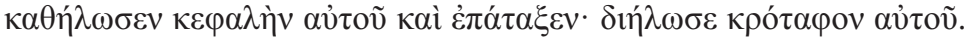

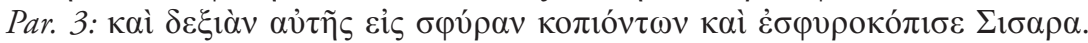

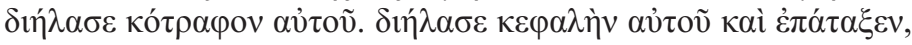

de la Políglota en otros libros bíblicos. Sí lo incluyen y son utilizados en nuestro estudio el Marc. 2 (= 29, partim s. IX, partim s. XIV), el Marc. 3 (=121, del s. XI), el Marc. 4 (=120, del s. XV), el Paris. 3 (= 56, del s. XI), el Marc. 5 (=68, del s. XV), el UCM 22 (= 442, copiado, según parece, en Venecia por Juan Severo de Lacedemonia a principios del s. XVI) y el Vatic. 330 (=108), del s. XIII y fuente de la Políglota en Jueces, además de las dos magnas ediciones principes: la Políglota, que se imprimió en Alcalá en julio de 1517, pero no se distribuyó hasta varios años después, y la Aldina, publicada en Venecia 1518-9 (Sáenz-Badillos, 1996: 139). "Son pues, dos ediciones casi contemporáneas, y el espíritu colaborativo y abierto que impregnó la empresa de la Políglota hace posible que existieran relaciones entre ambos procesos editoriales" (Hernández Muñoz, 2020: 232, n.10). En cuanto al Par. 3 (= 56), que también transmite Jueces, algunos investigadores, como M. Margolis, lo consideran modelo de la Políglota (cf. O’Connell, 2006: 15, n. 15 y 78 ss.; Fernández Marcos, 2014: 132-133; Gil Fernández, 2015: 294), cosa que nuestro estudio parece descartar, al menos en los pasajes estudiados.

${ }^{7}$ En cada pasaje pondremos siempre en primer lugar las dos versiones principales y más venerables del texto, la del Codex Alexandrinus $(A)$ y la del Vaticanus ( $V$. A continuación vendrán los testimonios colacionados que pueden tener relación con las ediciones Aldina (Al) y Políglota (Polig.): manuscritos UCM 22, Vat. 330, Par. 3 y Marc. 2, 3, 4, 5 y 6.

${ }^{8} \dot{\alpha} \chi \rho \varepsilon \imath \tilde{\omega} \sigma \alpha 1$ UCM 22, Marc. 2.

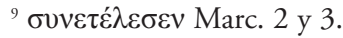

${ }^{10} \sum \varepsilon 1 \sigma \alpha ́ \rho \alpha$ Marc. 3.

${ }^{11} \delta$ ¿ฑ่ $\lambda \alpha \sigma \varepsilon v$ Marc. 2 у 3.

${ }^{12} \delta ı \eta ́ \lambda \alpha \sigma \varepsilon v$ Marc. 2 у 3.

${ }^{13} \kappa \alpha \tau \alpha \kappa о \pi \tau o ́ v \tau \omega v$ Polig. 
TEXTO 2 (Jueces, 5.27)

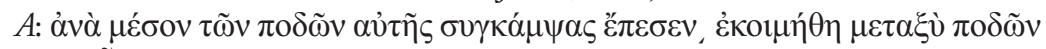

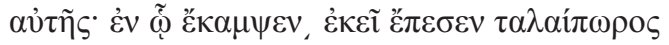

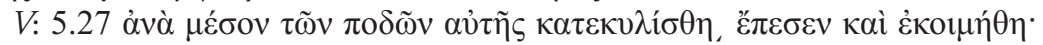

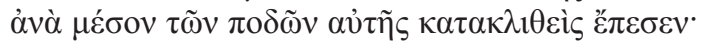

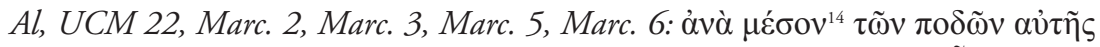

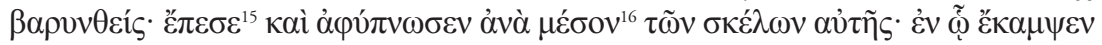

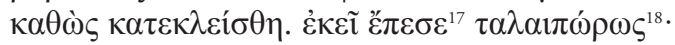

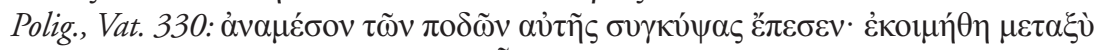

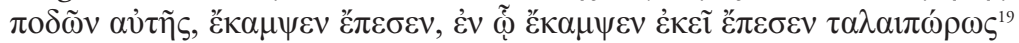

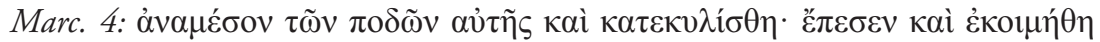

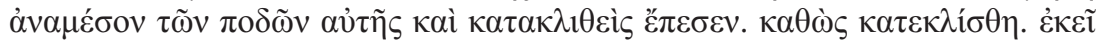

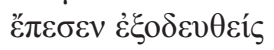

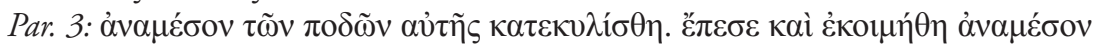

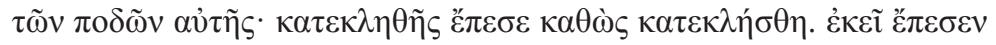

TEXTO 3 (Jueces, 5.30)

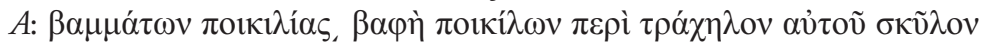

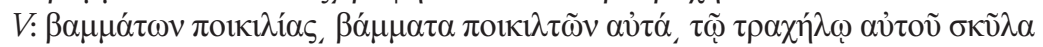

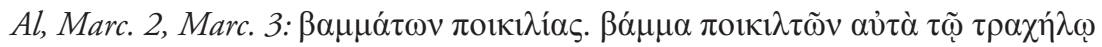

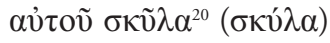

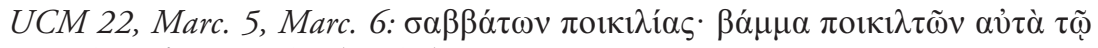

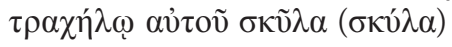

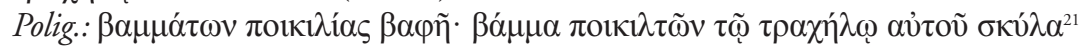

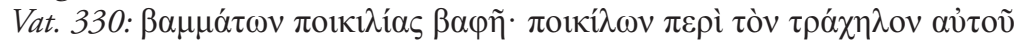

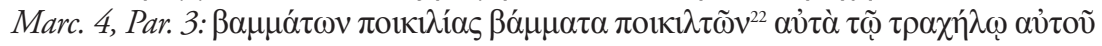
$\sigma \kappa \tilde{\lambda} \lambda \alpha(\sigma \kappa v ́ \lambda \alpha)$

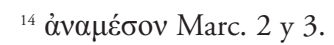

15 हैं $\pi \sigma \varepsilon v$ Marc. 2 у 3.

${ }_{16}^{16} \dot{\alpha} v \alpha \mu \varepsilon \dot{\varepsilon} \sigma o v$ Marc. 2 у 3.

${ }^{17} \ddot{\varepsilon} \pi \varepsilon \sigma \varepsilon v$ Marc. 2 y 3.

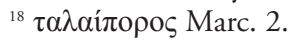

${ }^{19} \tau \alpha \lambda \alpha \imath \pi$ ó $\omega \varsigma$ Vat. 330.

${ }^{20}$ Este término sólo está acentuado así en la Aldina, el UCM 22 y el Marc. 4; en el resto de testimonios aparece sin acento (Marc. 2), con acento agudo (Marc. 3, 5, 6, Par. 3 y la Políglota) u omitida la palabra (Vat. 330).

${ }^{21}$ Pace O'Connell, (2006: 82), la Políglota presenta un texto que no coincide ni con el Marc. 5 ni con el UCM 22 ni con los otros testimonios colacionados (incluido su fuente principal en este libro, el Vat. 330, que, como vemos, además omite $\sigma \kappa \tilde{\lambda} \lambda \alpha$ ), fruto, quizá, del acceso a otro manuscrito todavía desconocido o de alguna intervención textual de los editores complutenses, pero es llamativa su cercanía con la Aldina y los Marc. 2 y 3.

${ }^{22} \pi$ oเкı $\lambda \iota \tilde{\omega} v$ Par. 3 (ut vid.). 


\section{CONCLUSIONES}

En el EJEMPLO 1 la Aldina parece presentar una conflatio, por un lado,

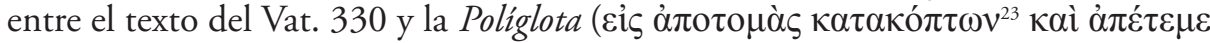

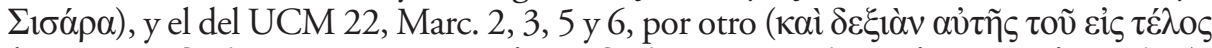

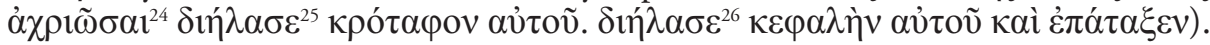

En el EJEMPLO 2 la Aldina sigue también este segundo patrón textual, apartándose del Vat 330 y la Políglota.

En el EJEMPLO 3 la Aldina sigue sólo el texto de los Marc. 2 y 3 , que coinciden también en algunas lecturas comunes.

En general, el texto del Vat. 330 y la Políglota se aproxima más a la versión del códice Alexandrinus $(A)$, mientras que el del Marc. 4 y el Paris. 3 se acerca más a la del Vaticanus ( $V$. El resto de testimonios (la Aldina, y los manuscritos UCM 22 y Marc. 2, 3, 5 y 6) suele presentar un texto más distante y mezclado de ambas versiones (quizá más de $V$ en el EJEMPLO 1 y 3 , y de $A$ en el 2).

Por tanto, confirmado el texto ecléctico de la edición Aldina, al menos en estos pasajes parece presentar una especial vinculación con los Marc. 2 y 3, lo que confirmaría la hipótesis de Sweete (1902: 174) y Fernández Marcos (2014: 126), pero sin excluir el acceso a otras fuentes ${ }^{27}$.

Y, por último, se confirma también, al menos en este libro bíblico ${ }^{28}$, la vinculación de la Políglota con el Vat. 330, del UCM 22 con el Marc. 5 (y el Marc. 6) ${ }^{29}$, y del Par. 3 con el Marc. 4.

\footnotetext{
${ }^{23} \kappa \alpha \tau \alpha \kappa o \pi \tau o ́ v \tau \omega v$ Polig

${ }^{24} \alpha \dot{\alpha} \chi \varepsilon \varepsilon 1 \tilde{\omega} \sigma \alpha 1$ Marc. 2.

${ }^{25} \delta$ เท́ं $\alpha \sigma \varepsilon v$ Marc. 2 y 3.

${ }^{26} \delta$ ¿ı́ $\lambda \alpha \sigma \varepsilon v$ Marc. 2 y 3
}

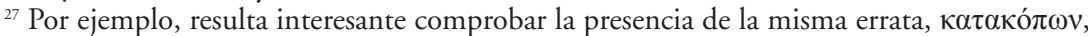
en el manuscrito Alexandrinus, en el 38 (= Scurial. Y.II.5) y en 15 (= Coislin. 2), según el aparato de BrookeMcLean y de Holmes-Parsons, respectivamente. Así también, en Macabeos II.9.21 la Aldina añade dos frases que no hallamos en los testimonios colacionados, pero sí, según Kappler-Hanhart (19594: 27) en la recensión $L$ (manuscritos 64, 236, 381, 534, 728) y la recensión $l$ (manuscritos 19, 62, 93, 542), es decir, en conjunto la recensión $L^{\prime}(L+l)$, a la que se añadirían los denominados "codices mixti" 46,

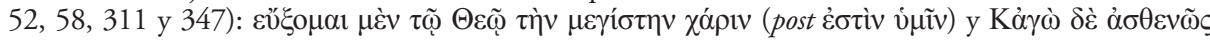

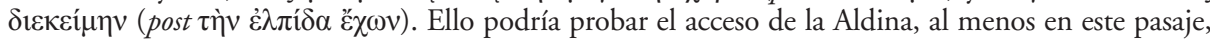
a otras fuentes.

${ }^{28}$ En otros libros la situación también cambia. Por ejemplo, hemos concluido (Hernández Muñoz, 2020: 245-246) que, en efecto, en el vol. II (en el que se halla Jueces) el Vat. 330 fue la fuente principal de la Polígota y, secundariamente, el UCM 22. Sin embargo, en el vol. III parece que la fuente primaria fue, por este orden, el Vat. 346 y el UCM 22, manuscrito este último que parece ser la principal en el vol. IV, sin descartar el acceso, directo o indirecto, a otros manuscritos, como el Vat. 348 (que transmite sólo los libros de Macabeos, ausentes en los otros dos Vaticanos -330 y 346- prestados por el papa León X a Cisneros) o, especialmente gracias a las anotaciones de Musuro, a los Marc. 1 y 4.

${ }^{29}$ Pero hemos comprobado que en Judith ambos Marcianos se separan más: el Marc. 6 suele coincidir con el Marc.1 (que no trasmite Jueces) y el Vat. 330, en tanto que el Marc. 5 suele hacerlo con el UCM 22 y el resto de testimonios, incluidas las ediciones Aldina y Políglota (en ocasiones 


\section{BIBLIOGRAFÍA}

ÁNGEL Y ESPINÓS, J. (2009): "El códice Complutensis Graecus 22: su destrucción y posterior recuperación”, en Actas del XII Congreso Español de Estudios Clásicos, vol. I, Sociedad Española de Estudios Clásicos, Madrid, pp. 177-184.

Bravo García, A. (2008): "UCM. Biblioteca Histórica Marqués de Valdecilla, Vill-Amil 22 (442 Rahlfs)”, en Lecturas de Bizancio. El legado escrito de Grecia en España. Biblioteca Nacional. Catálogo de la exposición, Biblioteca Nacional de España, Madrid, pp. 34-35.

Brooke, A. E. - McLean, M. A. (1914): The Old Testament in Greek, vol. I, Part IV, Cambridge University Press, Cambridge.

Cataldi Palau, A (1998): Gian Francesco D’Asola e la tipografia aldina, SAGEP, Genova.

DE ANDrÉs, G. (1974): "Catálogo de los códices griegos de las colecciones: Complutense, Lázaro Galdiano y March de Madrid”, Cuadernos de Filología Clásica 6: 239-249.

Delitzsch, F. (1886): Fortgesetzte Studien zur Entstehungsgeschichte der Complutensischen Poliglotte, Edelmann, Leipzig.

Domingo Malvadi, A. (2014): "Las fuentes de la Biblia Políglota en lengua griega”, en V Centenario de la Biblia Poliglota Complutense. La Universidad del Renacimiento. El Renacimiento de la Universidad. Catálogo de la exposición, UCM, Madrid, pp. 267-280 [la ficha del ms. UCM 22 en pp. 276-278 está redactada por J. L. GONZALO SÁNCHEZ-Molero y M. Torres SANTO DOMINGO].

FERnÁNDEZ MARCOS, N. (2005): "Un manuscrito complutense redivivo. Ms griego 442 = Villa-Amil 22", Sefarad 65: 65-83.

Fernández Marcos, N. (2014): "El texto griego de Septuaginta en la Políglota Complutense", en I. Carbajosa - A. García Serrano (eds.), Una Biblia a varias voces. Estudio textual de la Biblia Políglota Complutense, Ediciones Universidad San Dámaso, Madrid, pp. 125-142.

el Marc. 1 puede ubicarse en este segundo grupo). Aquí, en Jueces, los tres manuscritos (Marc. 5, Marc. 6 y UCM 22) coinciden estrechamente. La cercanía que hemos podido comprobar en todo el capítulo 5 de Jueces entre el texto de Gayangos y el Marc. 5 salvo en muy pocos pasajes (en primer lugar, la transcrip-

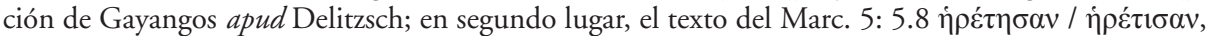

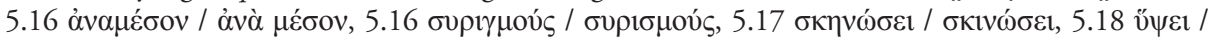

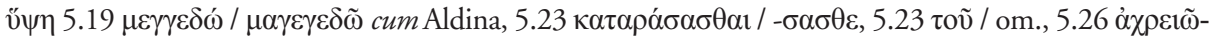

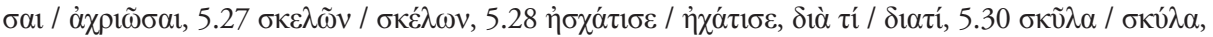
$5.31 \mathrm{\kappa aí} / \mathrm{om}$.) que pueden deberse a pequeños fallos en la transcripción o, lo que parece más probable, a mínimos cambios textuales entre ambos manuscritos por itacismos, "falso corte", etc., es prueba de que la transcripción de Gayangos de este capítulo, hoy perdido, de Jueces en el UCM 22 es lo suficientemente fidedigna como para que pueda ser utilizada en lugar del códice (instar codicis) en esos pasajes hoy perdidos. Por otra parte, en su transcripción Gayangos anota varios añadidos al margen

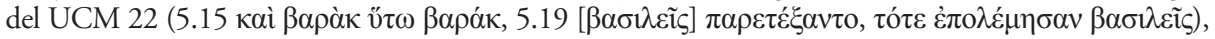
que hemos comprobado que se encuentran también en el Marc. 5 (y en el 6), lo que sugiere que, como ocurre con otros pasajes del UCM 22 donde esto ocurre (cf. Hernández Muñoz, 2020: 237, n. 25; 241-2, n. 44; 244, n. 51), corresponderían a anotaciones de Musuro que completaban el texto omitido, general-

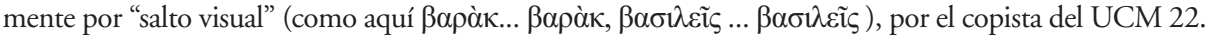


Gil Fernández, L. (2015): “A cuento del centenario del texto griego de la Poliglota Complutense”, Cuadernos de Filología Clásica (G) 25: 291-300.

Graux, Ch. - Martin, A. (1892): «Rapport sur une mission en Espagne et en Portugal. Notices sommaires des manuscrits grecs d'Espagne et de Portugal», Nouvelles archives des missions scientifiques et littéraires 2: 1-322.

Hernández Muñoz, F. G. (2020): "El texto griego de Septuaginta en la Biblia Políglota Complutense y su relación con otros testimonios, especialmente con el 'recuperado' manuscrito UCM (BH) 22”, Cuadernos de Filología Clásica (G) 30: 229-252.

Hanhart, R. (ed.) (1959): Maccabeorum Liber II, Academia Scientarum Gottingensis, Vandenhoeck \& Rupprecht, Göttingen.

Holmes, R. - Parsons, J. (eds.) (1818, 1827): Vetus Testamentum Graecum cum variis lectionibus, vol. II y V, University Press, Oxford.

KApPler, W. - Hanhart, R. (eds.) (1960²): Maccabeorum Liber III, Academia Scientarum Gottingensis, Vandenhoeck \& Rupprecht, Göttingen.

Lagarde, P. A. DE (ed.) (1868): Genesis Graece, Teubner, Leipzig.

LÓpez Rueda, J. (1973): Helenistas españoles del s. XVI, CSIC, Madrid.

Martínez Manzano, T. - Hernández Muñoz , F. G. (2019): “BH Mss. 22” y "BH Mss. 23”", en A. López FonseCa - M. Torres SANTo Domingo (eds.), Catálogo de manuscritos medievales de la Biblioteca Histórica "Marqués de Valdecilla (UCM)", Ediciones Complutense, Madrid, pp. 141-144.

MARTINS DE Jesus, C. (en prensa): "A incrível história de um manuscrito da Septuaginta quase destruído na Guerra Civil Espanhola (BH UCM 22 = 442 Rahlfs)”, en A. M. L. ANDrADE (ed.), Do Manuscrito ao Livro Impresso II, Imprensa da Universidade de Aveiro - Coimbra, Coimbra.

O'ConnelL, S. (2006): From most Ancient Sources: The Nature and Text-critical Use of the Greek Old Testament of the Complutensian Polyglot Bible, Academic Press-Vandenhoeck \& Ruprecht, FreiburgTübingen.

Rahlfs, A. (\& Hanhart, R) (1935'): Septuaginta I-II, Deutsche Bibelgesellschaft, Stuttgart.

Revilla Rico, M. (1917): La Políglota de Alcalá: estudio histórico-crítico, Imprenta helénica, Madrid.

SÁENZ-BADILlos, A. (1990): La filología bíblica en los primeros helenistas de Alcalá, Asociación Bíblica Española, Estella.

SÁenz-Badillos, A. (1996): "La Biblia Políglota Complutense”, en L. Jiménez Moreno (coord.), La Universidad Complutense Cisneriana. Impulso filosófico, científico y literario. Siglos XVI y XVII, Editorial Complutense, Madrid, pp. 137-153.

Speranzi, D. (2013): Marco Musuro. Libri e scrittura, Accademia Nazionale dei Lincei, Roma.

SweETe, H. B. (1902): An Introduction to the Old Testament in Greek, Cambridge University Press, Cambridge.

TAcón, J. - Puerto Manouvriez, P. (2001): "Códice griego del siglo XV semidestruido en la guerra civil. Montaje a partir de láminas de poliéster", Restauración y Rehabilitación. Revista internacional de Patrimonio Histórico 58: 70-74.

Torres Santo Domingo, M. (2005): "Libros que salvan vidas, libros que son salvados: La Biblioteca Universitaria en la Batalla de Madrid", en B. CALVo Alonso-CorTÉs (ed.), Biblioteca en Guerra. Catálogo de exposición, Biblioteca Nacional de España, Madrid, pp. 261-285.

Villa-Amil y CASTro, J. (1878): Catálogo de los manuscritos existentes en la Biblioteca del Noviciado de la Universidad Central (procedentes de la antigua de Alcalá). Parte I. Códices, Aribau y Ca, Madrid. 
